\title{
Association of a transcription factor 21 gene polymorphism with hypertension
}

\author{
TETSUO FUJIMAKI ${ }^{1}$, MITSUTOSHI OGURI ${ }^{2}$, HIDEKI HORIBE $^{3}$, KIMIHIKO KATO $^{4,5}$, \\ REIKO MATSUOKA ${ }^{6}$, SHINTARO ABE ${ }^{6}$, FUMITAKA TOKORO $^{6}$, MASAZUMI ARAI $^{6}$, \\ TOSHIYUKI NODA $^{6}$, SACHIRO WATANABE $^{6}$ and YOSHIJI YAMADA ${ }^{5}$
}

\begin{abstract}
${ }^{1}$ Department of Cardiovascular Medicine, Inabe General Hospital, Inabe, Mie 511-0428; ${ }^{2}$ Department of Cardiology, Japanese Red Cross Nagoya First Hospital, Nagoya, Aichi 453-8511; ${ }^{3}$ Department of Cardiovascular Medicine, Gifu Prefectural Tajimi Hospital, Tajimi, Gifu 507-8522; ${ }^{4}$ Department of Internal Medicine, Meitoh Hospital, Nagoya, Aichi 465-0025; ${ }^{5}$ Department of Human Functional Genomics, Life Science Research Center, Mie University, Tsu, Mie 514-8507; ${ }^{6}$ Department of Cardiology, Gifu Prefectural General Medical Center, Gifu, Gigu 500-8717, Japan
\end{abstract}

Received July 28, 2014; Accepted September 9, 2014

DOI: $10.3892 /$ br.2014.371

\begin{abstract}
Various loci and genes that confer susceptibility to coronary artery disease (CAD) have been identified mainly in Caucasian populations by genome-wide association studies (GWASs). As hypertension is a major risk factor for CAD, certain polymorphisms may contribute to the genetic susceptibility to CAD through affecting the predisposition to hypertension. The aim of the present study was to examine a possible association of hypertension with 29 single-nucleotide polymorphisms (SNPs) previously identified by meta-analyses of GWASs as susceptibility loci for CAD. Study subjects comprised of 5,460 individuals (3,348 subjects with hypertension and 2,112 controls). The genotypes of SNPs were determined by the multiplex bead-based Luminex assay. The $\chi^{2}$ test revealed that genotype distributions and allele frequencies for rs12190287 of the transcription factor 21 gene (TCF21) and rs1122608 of the SWI/SNF-related, matrix-associated, actin-dependent regulator of chromatin, subfamily a, member 4 gene (SMARCA4) were significantly $(\mathrm{P}<0.05)$ associated with hypertension. Allele frequencies for rs9369640 of the phosphatase and actin regulator 1 gene (PHACTRI) and genotype distributions for rs599839 of the proline/serine-rich coiled-coil 1 gene (PSRC1) were also significantly associated with hypertension. Multivariable logistic regression analysis with adjustment for age, gender, body mass index and smoking status revealed that rs12190287 of TCF 21 ( $\mathrm{P}=0.0014$; recessive model; odds ratio, 1.21) was significantly associated with hypertension, and
\end{abstract}

Correspondence to: Professor Yoshiji Yamada, Department of Human Functional Genomics, Life Science Research Center, Mie University, 1577 Kurima-machiya, Tsu, Mie 514-8507, Japan E-mail: yamada@gene.mie-u.ac.jp

Key words: hypertension, cardiovascular disease, genetics, polymorphism, $T C F 21$ the $C$ allele represented a risk factor for this condition. Similar analyses revealed that $\mathrm{rs} 1122608$ of SMARCA4 $(\mathrm{P}=0.0305$; dominant model; odds ratio, 0.86), rs9369640 of PHACTR1 $(\mathrm{P}=0.0119$; dominant model; odds ratio, 0.82) and rs599839 of PSRC1 ( $\mathrm{P}=0.0248$; dominant model; odds ratio, 0.84) were also related to hypertension, with the minor $T, C$ and $G$ alleles, respectively, being protective against this condition. Thus, the present results indicate that $\operatorname{rs} 12190287(G \rightarrow C)$ of $T C F 21$ is a susceptibility locus for hypertension.

\section{Introduction}

Hypertension remains a global public health problem, as it is an important risk factor for coronary artery disease (CAD), ischemic and hemorrhagic stroke and end-stage renal disease (1). Thus, prevention of hypertension may be an important strategy to reduce the overall burden of cardiovascular, cerebrovascular and renal diseases. Recent meta-analyses of genome-wide association studies (GWASs) for CAD or myocardial infarction in Caucasian populations identified various loci and genes that confer susceptibility to CAD $(2,3)$. As hypertension is a major risk factor of $\mathrm{CAD}$, certain polymorphisms may contribute to the genetic susceptibility to CAD through affecting the predisposition to hypertension. The aim of the present study was to examine a possible association of hypertension in Japanese individuals with 29 single-nucleotide polymorphisms (SNPs) previously identified by the meta-analyses of GWASs as susceptibility loci for CAD or myocardial infarction in Caucasian populations.

\section{Materials and methods}

Study population. Study subjects comprised of 5,460 Japanese individuals (3,348 subjects with hypertension and 2,112 controls) that visited outpatient clinics or were admitted to participating hospitals (Inabe General Hospital, Inabe; Gifu Prefectural General Medical Center, Gifu; Gifu Prefectural Tajimi Hospital, Tajimi; Japanese Red Cross Nagoya First Hospital, Nagoya; 
Hirosaki University Hospital and Hirosaki Stroke Center, Hirosaki, Japan) between 2002 and 2012. The subjects with hypertension had a systolic blood pressure (BP) of $\geq 140 \mathrm{mmHg}$ or a diastolic BP of $\geq 90 \mathrm{mmHg}$ (or both) or had taken antihypertensive medication. The control individuals had a systolic BP of $<140 \mathrm{mmHg}$ and a diastolic BP of $<90 \mathrm{mmHg}$ and no history of hypertension or of taking antihypertensive medication. BP was measured at least twice while subjects were relaxed in a sitting position for $>5 \mathrm{~min}$. The measurements were recorded by a skilled physician or a nurse according to the guidelines of the American Heart Association (4). The study protocol complied with the Declaration of Helsinki and was approved by the Ethics Committees in each participating hospital. Written informed consent was obtained from all the subjects.

Selection of polymorphisms. SNPs that were shown to be significantly associated with CAD or myocardial infarction were searched in Caucasian populations by the meta-analyses of GWASs $(2,3)$. These SNPs were examined with the dbSNP database (National Center for Biotechnology Information, http://www.ncbi.nlm.nih.gov/SNP/. Accessed March 3, 2013.) to find SNPs with a minor allele frequency of $\geq 0.015$ in a Japanese population. In total, 29 SNPs (data not shown) were finally selected and a possible association with hypertension was examined.

Genotyping of polymorphisms. Venous blood $(7 \mathrm{ml})$ was collected into tubes containing $50 \mathrm{mmol} / \mathrm{l}$ ethylenediaminetetraacetic acid (disodium salt), the peripheral blood leukocytes were isolated and genomic DNA was extracted from these cells with a DNA Extraction kit (Genomix; Talent Srl, Trieste, Italy). The SNP genotypes were determined at G\&G Science Co., Ltd., (Fukushima, Japan) by a method that combined polymerase chain reaction and sequence-specific oligonucleotide probes with suspension array technology (Luminex, Austin, TX, USA) as described previously (5-7). Detailed genotyping methodology was also described previously (8).

Statistical analysis. Quantitative data were compared between the subjects with hypertension and controls by the unpaired Student's t-test. Categorical data were compared by the $\chi^{2}$ test. Allele frequencies were estimated by the gene counting method. Departure from the Hardy-Weinberg equilibrium was examined by the $\chi^{2}$ test. Multivariable logistic regression analysis was performed with hypertension as a dependent variable, independent variables, including age, gender ( 0 , female; 1 , male), body mass index (BMI), smoking status (0, non-smoker; 1 , current or former smoker), and each genotype; and the P-value, odds ratio and $95 \%$ confidence interval were calculated. Genotypes of each polymorphism were assessed according to dominant (0, wild-type homozygote; 1 , heterozygote and variant homozygote), recessive (0, wild-type homozygote and heterozygote; 1 , variant homozygote) and additive genetic models. Additive models comprised additive 1 (heterozygotes vs. wild-type homozygotes) and additive 2 (variant homozygotes vs. wild-type homozygotes) models, which were analyzed simultaneously with a single statistical model. $\mathrm{P}<0.05$ was considered to indicate a statistically significant difference. Statistical test was performed with JMP version 5.1 and JMP Genomics version 6.0 software (SAS Institute, Inc., Cary, NC, USA).

\section{Results}

Patient characteristics. Characteristics of the 5,460 subjects are shown in Table I. Age, the frequency of males, BMI, the prevalence of smoking, ischemic stroke, CAD, diabetes mellitus and dyslipidemia, as well as serum concentrations of creatinine and triglycerides and fasting plasma glucose level were greater, whereas estimated glomerular filtration rate and serum concentrations of high-density lipoprotein-cholesterol were lower in the subjects with hypertension than in controls.

Comparisons of the genotype distributions or allele frequencies. On the basis of the genotype distribution or allele frequency comparisons by the $\chi^{2}$ test, 4 SNPs (including 2 of borderline significance) were associated with the prevalence of hypertension (Table II). The genotype distributions and allele frequencies for rs12190287 of the transcription factor 21 gene (TCF21) and rs1122608 of the SWI/SNF-related, matrix-associated, actin-dependent regulator of chromatin, subfamily a, member 4 gene (SMARCA4) were significantly $(\mathrm{P}<0.05)$ associated with the prevalence of hypertension. The allele frequencies for rs9369640 of the phosphatase and actin regulator 1 gene (PHACTR 1$)$ and genotype distributions for rs599839 of the proline/serine-rich coiled-coil 1 gene (PSRC1) were also significantly associated with hypertension. These SNPs with a $\mathrm{P}<0.05$ in genotype distributions or allele frequencies by the $\chi^{2}$ test were further examined by multivariable logistic regression analysis with adjustment for covariates.

Multivariable logistic regression analysis with adjustment for age, gender, BMI and smoking status revealed that rs12190287 of TCF21 (dominant, recessive and additive 2 models) was significantly $(\mathrm{P}<0.05)$ associated with hypertension, and the $C$ allele represented a risk factor for this condition (Table III). Similar analyses revealed that rs1122608 of SMARCA4, rs9369640 of PHACTR1 and rs599839 of PSRC1 (dominant and additive 1 models) were also associated with hypertension, with the minor $T, C$ and $G$ alleles, respectively, protecting against this condition.

Finally, the association of rs12190287, rs1122608, rs9369640 or rs599839 with systolic or diastolic BP was examined among all the individuals (Table IV). There were no significant differences in systolic or diastolic BP among the genotypes of these SNPs.

\section{Discussion}

Although GWASs have implicated various loci and genes in predisposition to hypertension in Caucasian (9-13) or African-Americans populations (14), the genes that confer susceptibility to this condition in the Japanese population remain to be definitively identified. The associations of the 29 SNPs identified by the meta-analyses of GWASs for CAD in Caucasian populations $(2,3)$ to hypertension were examined in 5,460 Japanese individuals. The large-scale association study revealed that $\mathrm{rs} 12190287(G \rightarrow C)$ of $T C F 21$ was significantly associated with the prevalence of hypertension in the Japanese population with the $C$ allele representing a risk factor for this condition. The rs1122608 of SMARCA4, rs9369640 of PHACTR1 and rs599839 of PSRC1 were also associated with hypertension, and the minor $T, C$ and $G$ alleles, respectively, were protective against this condition. 
Table I. Characteristics of 5,460 study subjects.

\begin{tabular}{|c|c|c|c|}
\hline Characteristics & Hypertension $(n=3,348)$ & Controls $(n=2,112)$ & P-values \\
\hline Age, years & $66.2 \pm 10.2$ & $61.9 \pm 12.1$ & $<0.0001$ \\
\hline Gender, men/women, $\%$ & $63.9 / 36.1$ & $55.0 / 45.0$ & $<0.0001$ \\
\hline Body mass index, $\mathrm{kg} / \mathrm{m}^{2}$ & $24.0 \pm 3.4$ & $23.5 \pm 2.9$ & $<0.0001$ \\
\hline Current or former smoker, $\%$ & 25.5 & 21.7 & 0.0017 \\
\hline Ischemic stroke, $\%$ & 20.1 & 10.5 & $<0.0001$ \\
\hline Coronary artery disease, $\%$ & 54.2 & 30.6 & $<0.0001$ \\
\hline Systolic blood pressure, $\mathrm{mmHg}$ & $151.6 \pm 25.5$ & $120.4 \pm 12.7$ & $<0.0001$ \\
\hline Diastolic blood pressure, $\mathrm{mmHg}$ & $80.8 \pm 16.0$ & $69.1 \pm 10.0$ & $<0.0001$ \\
\hline Serum creatinine, $\mu \mathrm{mol} / 1$ & $98.8 \pm 126.5$ & $71.0 \pm 26.7$ & $<0.0001$ \\
\hline Estimated GFR, ml/min/1.73 $\mathrm{m}^{2}$ & $65.8 \pm 24.9$ & $73.7 \pm 22.1$ & $<0.0001$ \\
\hline Diabetes mellitus, $\%$ & 43.8 & 21.4 & $<0.0001$ \\
\hline Fasting plasma glucose, $\mathrm{mmol} / \mathrm{l}$ & $7.41 \pm 3.48$ & $6.40 \pm 3.17$ & $<0.0001$ \\
\hline Dyslipidemia, $\%$ & 51.3 & 30.1 & $<0.0001$ \\
\hline Serum triglycerides, mmol/l & $1.70 \pm 1.13$ & $1.51 \pm 1.21$ & $<0.0001$ \\
\hline Serum HDL-cholesterol, mmol/1 & $1.28 \pm 0.38$ & $1.39 \pm 0.41$ & $<0.0001$ \\
\hline Serum LDL-cholesterol, mmol/1 & $3.12 \pm 0.93$ & $3.08 \pm 0.90$ & 0.1592 \\
\hline
\end{tabular}

Quantitative data are expressed as mean \pm standard deviation. Hypertension, systolic blood pressure of $\geq 140 \mathrm{mmHg}$, diastolic blood pressure of $\geq 90 \mathrm{mmHg}$, or taking antihypertensive medication; diabetes mellitus, fasting plasma glucose concentration of $\geq 6.93 \mathrm{mmol} / 1 \mathrm{or}$ taking antidiabetic medication; dyslipidemia, serum concentration of triglycerides of $\geq 1.65 \mathrm{mmol} / \mathrm{l}$, serum concentration of high-density lipoprotein (HDL)-cholesterol of $<1.04 \mathrm{mmol} / \mathrm{l}$, serum concentration of low-density lipoprotein (LDL)-cholesterol of $\geq 3.64 \mathrm{mmol} / \mathrm{l}$, or taking antidyslipidemic medication; estimated glomerular filtration rate $(\mathrm{GFR})\left(\mathrm{ml} / \mathrm{min} / 1.73 \mathrm{~m}^{2}\right)=194 \mathrm{x}$ [age (years) $]^{-0.287} \mathrm{x}$ [serum creatinine $\left.(\mathrm{mg} / \mathrm{dl})\right]^{-1.094} \mathrm{x}(0.739$ when female) .

Table II. Comparisons of genotype distributions and allele frequencies of rs12190287, rs1122608, rs9369640 or rs599839 by the $\chi^{2}$ test between the subjects with hypertension and controls.

\begin{tabular}{|c|c|c|c|c|c|c|}
\hline Gene & Polymorphism & dbSNP & Hypertension, \% & Controls, $\%$ & $\mathrm{P}$ (genotype) & $\mathrm{P}$ (allele) \\
\hline \multirow[t]{5}{*}{$T C F 21$} & $G \rightarrow C$ & rs 12190287 & & & 0.0132 & 0.0032 \\
\hline & $G G$ & & $563(16.8)$ & $402(19.0)$ & & \\
\hline & $G C$ & & 1595 (47.6) & 1033 (48.9) & & \\
\hline & $C C$ & & $1190(35.5)$ & $677(32.1)$ & & \\
\hline & Hardy-Weinberg P & & 0.4674 & 0.8215 & & \\
\hline \multirow[t]{5}{*}{ SMARCA4 } & $G \rightarrow T$ & rs1122608 & & & 0.0442 & 0.0143 \\
\hline & $G G$ & & $2636(78.8)$ & $1601(75.9)$ & & \\
\hline & $G T$ & & $674(20.1)$ & $481(22.8)$ & & \\
\hline & $T T$ & & $37(1.1)$ & $28(1.3)$ & & \\
\hline & Hardy-Weinberg P & & 0.7006 & 0.2267 & & \\
\hline \multirow[t]{5}{*}{ PHACTR1 } & $A \rightarrow C$ & rs9369640 & & & 0.0572 & 0.0161 \\
\hline & $A A$ & & $2825(84.5)$ & $1730(82.1)$ & & \\
\hline & $A C$ & & $494(14.8)$ & 357 (16.9) & & \\
\hline & $C C$ & & $25(0.7)$ & $21(1.0)$ & & \\
\hline & Hardy-Weinberg P & & 0.5057 & 0.5901 & & \\
\hline \multirow[t]{5}{*}{ PSRC1 } & $A \rightarrow G$ & rs599839 & & & 0.0382 & 0.0681 \\
\hline & $A A$ & & $2853(85.8)$ & $1761(83.6)$ & & \\
\hline & $A G$ & & $446(13.4)$ & $332(15.8)$ & & \\
\hline & $G G$ & & $26(0.8)$ & $12(0.6)$ & & \\
\hline & Hardy-Weinberg P & & 0.0657 & 0.3902 & & \\
\hline
\end{tabular}

dbSNP, the single nucleotide polymorphism database; TCF21, transcription factor 21; Hardy-Weinberg P, a P-value of Hardy-Weinberg equilibrium; SMARCA4, SWI/SNF-related, matrix-associated, actin-dependent regulator of chromatin, subfamily a, member 4; PHACTR1, phosphatase and actin regulator 1; PSRC1, proline/serine-rich coiled-coil 1. 
Table III. Multivariable logistic regression analysis for the association of rs12190287, rs1122608, rs9369640 or rs599839 with hypertension with adjustment for age, gender, body mass index and smoking status.

\begin{tabular}{|c|c|c|c|c|c|c|c|}
\hline \multirow[b]{2}{*}{ Gene } & \multirow[b]{2}{*}{ Polymorphism } & \multicolumn{2}{|r|}{ Dominant } & Recessive & \multicolumn{2}{|c|}{ Additive 1} & Additive 2 \\
\hline & & P-value & OR $(95 \% \mathrm{CI})$ & P-value OR $(95 \% \mathrm{CI})$ & P-value & OR $(95 \% \mathrm{CI})$ & P-value OR $(95 \% \mathrm{CI})$ \\
\hline TCF 21 & rs12190287 $(G \rightarrow C)$ & $0.0168^{a}$ & $1.20(1.03-1.38)$ & $0.0014^{\mathrm{a}} 1.21(1.08-1.37)$ & 0.1620 & & $0.0010^{\mathrm{a}} 1.32(1.12-1.55)$ \\
\hline SMARCA4 & $\operatorname{rs} 1122608(G \rightarrow T)$ & $0.0305^{\mathrm{a}}$ & $0.86(0.75-0.99)$ & 0.7103 & $0.0330^{\mathrm{a}}$ & $0.86(0.75-0.99)$ & 0.6202 \\
\hline PHACTRI & rs9369640 $(A \rightarrow C)$ & $0.0119^{\mathrm{a}}$ & $0.82(0.71-0.96)$ & 0.2908 & $0.0190^{\mathrm{a}}$ & $0.83(0.71-0.97)$ & 0.2497 \\
\hline PSRC1 & rs599839 $(A \rightarrow G)$ & $0.0248^{\mathrm{a}}$ & $0.84(0.72-0.98)$ & 0.2232 & $0.0110^{\mathrm{a}}$ & $0.81(0.69-0.95)$ & 0.2567 \\
\hline
\end{tabular}

${ }^{a} \mathrm{P}<0.05$. OR, odds ratio; CI, confidence interval; TCF 21, transcription factor 21; SMARCA4, SWI/SNF-related, matrix-associated, actin-dependent regulator of chromatin, subfamily a, member 4; PHACTR1, phosphatase and actin regulator 1; PSRC1, proline/serine-rich coiled-coil 1.

Table IV. Association of systolic or diastolic blood pressure (BP) with the TCF21, SMARCA4, PHACTR1 or PSRC1 genotypes in all the subjects.

\begin{tabular}{|c|c|c|c|c|c|c|}
\hline \multirow{2}{*}{$\begin{array}{l}\text { Variables } \\
T C F 21 \text { (rs12190287) }\end{array}$} & \multicolumn{3}{|c|}{ Genotype } & \multirow[t]{2}{*}{ P (ANOVA) } & \multirow[t]{2}{*}{$\mathrm{P}$ (dominant) } & \multirow[t]{2}{*}{$\mathrm{P}($ recessive $)$} \\
\hline & $G G$ & $G C$ & $C C$ & & & \\
\hline No. of subjects & 687 & 1916 & 1344 & & & \\
\hline Systolic BP, mmHg & $143 \pm 25$ & $143 \pm 27$ & $144 \pm 27$ & 0.8477 & 0.8928 & 0.5673 \\
\hline Diastolic BP, mmHg & $77 \pm 16$ & $78 \pm 15$ & $78 \pm 16$ & 0.5614 & 0.4006 & 0.3632 \\
\hline SMARCA4 (rs1122608) & $G G$ & $G T$ & $T T$ & & & \\
\hline No. of subjects & 3056 & 840 & 43 & & & \\
\hline Systolic BP, mmHg & $144 \pm 27$ & $143 \pm 27$ & $141 \pm 24$ & 0.8213 & 0.6111 & 0.6456 \\
\hline Diastolic BP, mmHg & $78 \pm 15$ & $78 \pm 16$ & $77 \pm 15$ & 0.9333 & 0.8666 & 0.7701 \\
\hline PHACTR1 (rs9369640) & $A A$ & $A C$ & $C C$ & & & \\
\hline No. of subjects & 3340 & 569 & 26 & & & \\
\hline Systolic BP, mmHg & $143 \pm 27$ & $144 \pm 26$ & $143 \pm 30$ & 0.9155 & 0.6785 & 0.9906 \\
\hline Diastolic BP, mmHg & $78 \pm 16$ & $77 \pm 14$ & $76 \pm 12$ & 0.3185 & 0.1406 & 0.5346 \\
\hline PSRC1 (rs599839) & $A A$ & $A G$ & $G G$ & & & \\
\hline No. of subjects & 3363 & 523 & 29 & & & \\
\hline Systolic BP, mmHg & $143 \pm 26$ & $144 \pm 28$ & $147 \pm 24$ & 0.8109 & 0.7541 & 0.5351 \\
\hline Diastolic BP, mmHg & $78 \pm 15$ & $78 \pm 16$ & $76 \pm 11$ & 0.7536 & 0.8679 & 0.4957 \\
\hline
\end{tabular}

$T C F 21$, transcription factor 21; SMARCA4, SWI/SNF-related, matrix-associated, actin-dependent regulator of chromatin, subfamily a, member 4; PHACTR1, phosphatase and actin regulator 1; PSRC1, proline/serine-rich coiled-coil 1; ANOVA, analysis of variance.

TCF21 is a class II basic helix-loop-helix transcription factor, which is expressed in the mesenchyme of developing organs, including lung, kidney and epicardium (15). TCF21 binds specific DNA sequences and negatively regulates various cell differentiation through modulating cell cycle arrest and tissue-specific gene expression (15), indicating that TCF 21 has a role in the development of malignant tumors (16). Expression quantitative trait locus (eQTL) data and a reporter gene assay showed that the $C$ allele of rs12190287 increased the expression of TCF21 in vascular smooth muscle cells (VSMCs) $(2,17)$. An in vitro study of cultured MG63 cells showed that expression of the cyclin-dependent kinase inhibitor, $P 21$, was reduced when $T C F 21$ was introduced (15), indicating that TCF21 functions as a negative regulator of $P 21$, which is crucial in VSMC proliferation (18). As microvascular structural narrowing caused by aberrant proliferation of VSMCs may play an important role in the development of hypertension (19), altered TCF 21 expression may affect BP trait through $P 21$-dependent microvascular remodeling.

SMRACA4 is located adjacent to the low-density lipoprotein receptor gene and regulates the transcription of various genes through disrupting chromatin structure using the chemical energy of adenosine triphosphate hydrolysis (20). Previous eQTL data indicated that the expression of SMARCA4 is likely modulated by rs1122608 (2). A possible regulating role of SMARCA4 in P21 expression and in VSMCs differentiation through myocardin-mediated pathway has also been reported $(21,22)$.

PHACTRl is expressed predominantly in neuronal tissues in brain (23). Although the precise function of PHACTRI remains unclear, associations with cell migration, motility and invasiveness in tumor tissues have been reported (24). 
PHACTR 1 may affect the activity of phosphatase 1, which is indicated to be involved in the regulation of nitric oxide synthesis in endothelial cells (25), through modulating G-actin binding affinity to its RPEL motif (23).

PSRC1 is a microtubules-associated protein that directly binds microtubules and controls the density, assembly and dynamics of microtubules, indicating that it plays a key role in the regulation of chromosome congression and segregation during mitosis (26). PSRC1 was also shown to be a susceptibility locus for a plasma concentration of low-density lipoprotein-cholesterol in a previous GWAS (27).

Although 4 SNPs were significantly associated with the prevalence of hypertension, genotypes of these polymorphisms were not associated with systolic or diastolic BP among all the subjects. These results did not change when untreated hypertensive subjects or control individuals were examined separately (data not shown). Although the reason for this discrepancy remains unclear, there were certain possibilities: i) The number of hypertensive subjects not taking antihypertensive medication was small; ii) information for drug compliance obtained by a questionnaire was incomplete; and iii) a substantial proportion of the subjects had white-coat hypertension.

The present study had several limitations: i) As the results of the present study were not replicated, validation of the findings is required in other independent subject panels or in other ethnic groups; ii) it is possible that 4 SNPs identified in the present study are in linkage disequilibrium with other polymorphisms in the same gene or in other nearby genes that are actually responsible for the development of hypertension; and iii) the functional relevance of rs12190287, rs1122608, rs9369640 or rs599839 to pathogenesis of hypertension remains to be elucidated.

In conclusion, $\mathrm{rs} 12190287(G \rightarrow C)$ of $T C F 21$ may be a susceptibility locus for hypertension in Japanese individuals. The rs1122608 of SMARCA4, rs9369640 of PHACTR1 and rs599839 of PSRC1 were also associated with hypertension. Determination of genotypes of these SNPs may prove informative for assessment of the genetic risk for hypertension in Japanese individuals.

\section{Acknowledgements}

The present study was supported by a Collaborative Research Grant from the Gifu Prefectural General Medical Center (no. H24-26 to Y.Y.) and a Grant-in-Aid for Scientific Research from the Ministry of Education, Culture, Sports, Science and Technology of Japan (no. 24590746 to Y.Y.).

\section{References}

1. Kearney PM, Whelton M, Reynolds K, et al: Global burden of hypertension: analysis of worldwide data. Lancet 365: 217-223, 2005.

2. Schunkert H, Konig IR, Kathiresan S, et al: Large-scale association analysis identifies 13 new susceptibility loci for coronary artery disease. Nat Genet 43: 333-338, 2011.

3. CARDIoGRAMplusC4D Consortium, Deloukas P, Kanoni S, et al: Large-scale association analysis identifies new risk loci for coronary artery disease. Nat Genet 45: 25-33, 2013.

4. Perloff D, Grim C, Flack J, Frohlich ED, Hill M, McDonald M and Morgenstern BZ: Human blood pressure determination by sphygmomanometry. Circulation 88: 2460-2470, 1993.
5. Yamada Y, Fuku N, Tanaka M, et al: Identification of CELSR1 as a susceptibility gene for ischemic stroke in Japanese individuals by a genome-wide association study. Atherosclerosis 207: 144-149, 2009.

6. Yamada Y, Nishida T, Ichihara S, et al: Association of a polymorphism of BTN2A1 with myocardial infarction in East Asian populations. Atherosclerosis 215: 145-152, 2011.

7. Yamada Y, Nishida T, Ichihara S, et al: Identification of chromosome 3q28 and ALPK1 as susceptibility loci for chronic kidney disease in Japanese individuals by a genome-wide association study. J Med Genet 50: 410-418, 2013.

8. Itoh Y, Mizuki N, Shimada T, et al: High-throughput DNA typing of HLA-A, -B, -C, and -DRB1 loci by a PCR-SSOP-Luminex method in the Japanese population. Immunogenetics 57: 717-729, 2005.

9. Wellcome Trust Case Control Consortium: Genome-wide association study of 14,000 cases of seven common diseases and 3,000 shared controls. Nature 447: 661-678, 2007.

10. Newton-Cheh C, Johnson T, Gateva V, et al: Genome-wide association study identifies eight loci associated with blood pressure. Nat Genet 41: 666-676, 2009.

11. Levy D, Ehret GB, Rice K, et al: Genome-wide association study of blood pressure and hypertension. Nat Genet 41: 677-687, 2009.

12. Org E, Eyheramendy S, Juhanson P, et al: Genome-wide scan identifies $\mathrm{CDH} 13$ as a novel susceptibility locus contributing to blood pressure determination in two European populations. Hum Mol Genet 18: 2288-2296, 2009.

13. Tragante V, Barnes MR, Ganesh SK, et al: Gene-centric meta-analysis in 87,736 individuals of European ancestry identifies multiple blood-pressure-related loci. Am J Hum Genet 94: 349-360, 2014

14. Adeyemo A, Gerry N, Chen G, et al: A genome-wide association study of hypertension and blood pressure in African Americans. PLoS Genet 5: e1000564, 2009.

15. Funato N, Ohyama K, Kuroda $\mathrm{T}$ and Nakamura M: Basic helix-loop-helix transcription factor epicardin/capsulin/Pod-1 suppresses differentiation by negative regulation of transcription. J Biol Chem 278: 7486-7493, 2003.

16. Smith LT, Lin M, Brena RM, et al: Epigenetic regulation of the tumor suppressor gene TCF21 on 6q23-q24 in lung and head and neck cancer. Proc Natl Acad Sci USA 103: 982-987, 2006.

17. Miller CL, Anderson DR, Kundu RK, et al: Disease-related growth factor and embryonic signaling pathways modulate an enhancer of TCF21 expression at the 6q23.2 coronary heart disease locus. PLoS Genet 9: e1003652, 2013.

18. Bond M, Sala-Newby GB, Wu YJ and Newby AC: Biphasic effect of p21Cip1 on smooth muscle cell proliferation: role of PI 3-kinase and Skp2-mediated degradation. Cardiovasc Res 69: 198-206, 2006

19. Feihl F, Liaudet L, Levy BI and Waeber B: Hypertension and microvascular remodelling. Cardiovasc Res 78: 274-285, 2008.

20. Peterson CL: Chromatin remodeling: nucleosome bulging at the seams. Curr Biol 12: R245-R247, 2002.

21. Zhou J, Zhang M, Fang H, et al: The SWI/SNF chromatin remodeling complex regulates myocardin-induced smooth muscle-specific gene expression. Arterioscler Thromb Vasc Biol 29: 921-928, 2009.

22. Hendricks KB, Shanahan F and Lees E: Role for BRG1 in cell cycle control and tumor suppression. Mol Cell Biol 24: 362-376, 2004.

23. Allen PB, Greenfield AT, Svenningsson P, Haspeslagh DC and Greengard P: Phactrs 1-4: A family of protein phosphatase 1 and actin regulatory proteins. Proc Natl Acad Sci USA 101: 7187-7192, 2004.

24. Fils-Aime N, Dai M, Guo J, El-Mousawi M, Kahramangil B, Neel JC and Lebrun JJ: MicroRNA-584 and the protein phosphatase and actin regulator 1 (PHACTR1), a new signaling route through which transforming growth factor- $\beta$ mediates the migration and actin dynamics of breast cancer cells. J Biol Chem 288: 11807-11823, 2013.

25. Mount PF, Kemp BE and Power DA: Regulation of endothelial and myocardial NO synthesis by multi-site eNOS phosphorylation. J Mol Cell Cardiol 42: 271-279, 2007.

26. Jang CY and Fang G: The N-terminal domain of DDA3 regulates the spindle-association of the microtubule depolymerase Kif $2 \mathrm{a}$ and controls the mitotic function of DDA3. Cell Cycle 8: 3165-3171, 2009.

27. Kathiresan S, Melander O, Guiducci C, et al: Six new loci associated with blood low-density lipoprotein cholesterol, high-density lipoprotein cholesterol or triglycerides in humans. Nat Genet 40: 189-197, 2008. 